\title{
DOMINANCIA CEREBRAL ASOCIADA AL DESEMPEÑO LABORAL DE LOS DOCENTES DE UNA UGEL DE LIMA
}

\author{
CEREBRAL DOMINANCE ASSOCIATED WITH THE LABOUR \\ PERFORMANCE OF TEACHERS IN A UGEL OF LIMA
}

\author{
Manuel Torres V. ${ }^{1}$, Rosario Lajo L. \\ Universidad Nacional Mayor de San Marcos \\ (Recibido el 4/04/2009, Aceptado el 17/06/2009)
}

\begin{abstract}
RESUMEN
Los avances en las neurociencias de la educación ponen de relieve la gran importancia que tiene desarrollar el cerebro del hombre para lograr su completo funcionamiento y hacerlo altamente productivo. Este desarrollo del cerebro debe empezar en la familia y continuarse muy activamente en la escuela como una de sus tareas centrales y el responsable de este trabajo es el docente, razón por la cual es una tarea impostergable desarrollar investigaciones y luego formular programas que capaciten al maestro en estas tareas. El objetivo central de este estudio fue identificar los cuadrantes cerebrales predominantes y las características del desempeño laboral en una muestra de docentes de una UGEL de Lima, para luego correlacionarlos entre sí.
\end{abstract}

Los instrumentos que se utilizaron fueron: el Inventario de Dominancia cerebral de RuizBolívar y colaboradores y el cuestionario de Autorreporte del desempeño laboral docente de Fernández Arata. Estos instrumentos fueron sometidos a los análisis respectivos que determinaron que las pruebas son validas y confiables.

La muestra estuvo constituida por 251 docentes de una UGEL de Lima, de los cuales 118 fueron varones y 133 mujeres, a quienes se les aplicó los cuestionarios indicados y luego se procedió a correlacionarlos. Los resultados indican que existen correlaciones significativas y positivas entre la dominancia cerebral y el desempeño laboral de los docentes, igualmente se encontró que un alto porcentaje de docentes que no tienen dominancia cerebral definida y que en general entre los docentes predomina el cerebro límbico y particularmente el cuadrante cerebral $\mathrm{B}$, aquel destinado a cumplir programaciones previamente planificadas sin ánimos de cambio.

Palabras clave: Cerebro, Neurociencias, dominancia cerebral, modos de pensamiento, desempeño laboral, educación.

1 Docente investigador del Instituto de Investigaciones Psicológicas de la Facultad de Psicología, Universidad Nacional Mayor de San Marcos, Lima-Perú. E-mail: mtorresv@unmsm.edu.pe 


\begin{abstract}
The progresses in neurosciences of the education put into spotlight the great importance that the human brain has to develop in order to obtain its full operation and make it highly productive. This development of the brain must start in the family and continued actively in the school as one of the main task and the responsible of this work is the teacher that is

why the development of investigations and the creation of programmes that capacitate the teacher are task that cannot be postponed. The main purpose of this study is to identify the predominant cerebral quadrants and the characteristics of the labour development in a sample of teachers from Lima's UGEL, and correlate them later.

The instruments utilised were: the Inventory of Cerebral Dominance of Ruiz-Bolívar and collaborators and the questionnaire of self-report of teacher's labour development of Fernández Arata. These instruments were taken into the respective analysis that showed the tests are valid and trustable.

The sample were constituted by 251 teachers of Lima's UGEL, from whom 118 were men and 133 women, to whom the indicated questionnaires were taken and they were later correlated. The results show that significant and positive correlations exist between the cerebral dominance and the labour development of teacher. It was also found that a high percentage of teachers that do not have defined cerebral dominance and that in general, the limbic brain predominate in the teachers, especially the cerebral quadrant B, the one destined to fulfil programmes previously planned without moods changes.
\end{abstract}

Keywords: Brain, neurosciences, cerebral dominance, ways of thoughts, labour development, education.

\title{
INTRODUCCIÓN
}

Que la Educación desempeña un papel de primer orden en el desarrollo económicosocial de los diferentes países en el mundo, es un tema que ya nadie discute. Hoy día el reconocimiento de este hecho hace que los gobiernos estén interesados en realizar todas las reformas educativas que sean necesarias a fin de garantizar una educación de calidad y que esté en relación directa a las exigencias que plantea el mundo globalizado.

Pero una educación de calidad pasa necesariamente por reconocer el papel de primer orden que tienen los profesores en todo el sistema educativo. Pero, realmente, ¿esto es así? De acuerdo a Díaz y Saavedra (2000), el maestro peruano es el que menores ingresos tiene en relación a otras profesionales, más aún no existen posibilidades reales de mejorar su situación económica en el corto plazo, a ello se suman las dificultades tan grandes que existen en su proceso de formación profesional, las dificultades de capacitación, los pocos incentivos para realizar estudios de posgrado, etc.

En estas condiciones el trabajo del maestro presenta grandes limitaciones lo que ha generado un conjunto de críticas, particularmente de los sectores gubernamentales, quienes pretenden responsabilizarlos de la cuestionada calidad educativa que se observa regularmente en las instituciones educativas, razón por las que se han desarrollados procesos de evaluación docente. Sin embargo, todas las evaluaciones que se han propuesto consideran en lo fundamental la actividad práctica del maestro en su desempeño en el aula de clase y se ha descuidado las condiciones internas que generan esta actividad práctica y que se refieren por ejemplo al funcionamiento del cerebro. 
El acelerado desarrollo de las neurociencias sugiere que los diversos resultados de las investigaciones sobre el cerebro permiten mayor comprensión de su funcionamiento, y ello debe necesariamente ser incorporado a los estudios sobre el mejoramiento de la habilidad del docente para enseñar y la habilidad del estudiante para aprender, y consecuentemente sus resultados permitirán construir una nueva pedagogía, pues el espacio de la pedagogía de hoy, es un espacio de la mente y de los fenómenos mentales.

Contrariamente, la educación de los estudiantes no se dirige al sistema nervioso, pues los teóricos de la educación, generalmente, no teorizan sobre el cerebro y los procesos cerebrales del estudiante; los escenarios del futuro exigen la construcción de esta nueva pedagogía orientada hacia la innovación y la creatividad, nacida de la interconexión entre la pedagogía y las neurociencias; en ella dos cuestiones serán consideradas claves: la filogenia y la ontogenia de la inteligencia, y la importancia de la estimulación y el aprendizaje temprano. Los avances de la neurociencia han ampliado los límites del espacio pedagógico, más allá de una simple expansión, pues habrá la necesidad de definir no solo los límites de ese espacio pedagógico sino los límites de la educación.

Resulta preocupante por eso que todos estos avances en las investigaciones sobre el cerebro no puedan ser puestos en ejercicio en el campo educativo, preocupación que se profundiza frente a la proliferación de prácticas docentes inadecuadas que crean climas o atmósferas que dificultan el proceso de aprendizaje y el desarrollo de la creatividad, el cultivo, casi exclusivo, de un solo hemisferio, el izquierdo, y de sus funciones racionales conscientes, descuidando la intuición y las funciones holísticas y gestálticas del derecho, marginando el componente emotivo y afectivo, la evaluación escolar inhibidora que coarta potencialidades, iniciativas e impulsos del estudiante que lo proyecta más allá de los objetivos que se le han programado desde afuera, la imposición de una normativa metodológica que lo reduce a caminar por donde ya se caminó, a explorar como antes se exploró, a pensar como antes se pensó; es decir, a no hacer nada que no se haya hecho antes.

El reconocimiento de estos hechos desafía un sistema educativo que supone que todos pueden aprender las mismas cosas del mismo modo, y que basta con una medida uniforme y universal para poner a prueba a un estudiante; nada más alejado de la realidad. En general el trabajo del maestro no puede estar encaminado a transmitir conocimientos, hoy debe preocuparse, considerando los avances de las neurociencias y en particular los trabajos de Herrmann, por el desarrollo global del cerebro de sus alumnos.

La presente investigación se ha desarrollado bajo la consideración de aportar en el proceso de construcción de un nuevo paradigma educacional, con una base epistemológica que nace de la neurofisiología y que permita, desde esta nueva perspectiva, explicar las teorías de aprendizaje y en general del proceso de aprendizaje del estudiante sin obviar, claro está, a todas las disciplinas que intervienen en este proceso.

Los avances en la investigación cerebral no pueden seguir siendo ignorados por la Educación por ello necesariamente deben ser incorporados al desarrollo de los estudios sobre las condiciones del aprendizaje y de la relación profesor-estudiante. Estamos convencidos que los resultados alcanzados permitirán construir una nueva pedagogía que considere de manera fundamental el órgano responsable del proceso de aprendizaje de los seres humanos: El cerebro. 
Como consecuencia de lo expuesto, los problemas de la presente investigación se plantean de la siguiente manera:

- ¿Qué relaciones existen entre la dominancia cerebral y el desempeño laboral de los docentes de una UGEL de Lima?

- ¿Qué diferencias existen entre los docentes varones y mujeres de una UGEL de Lima respecto de las Escalas de dominancia cerebral?

- ¿Qué diferencias existen entre los docentes varones y mujeres de la UGEL No 7 Lima respecto de las Escalas de desempeño laboral?

- ¿Qué diferencias existen entre los docentes provenientes de universidades e institutos pedagógicos de una UGEL de Lima respecto de las escalas de dominancia cerebral?

- ¿Qué diferencias existen entre los docentes provenientes de Universidades e institutos pedagogicos de una UGEL de Lima respecto de las escalas de desempeño laboral?

- ¿Cuál es el tipo de dominancia cerebral predominante entre los docentes de una UGEL de Lima?

- ¿Cuál es el cuadrante cerebral predominante entre los docentes de una UGEL de Lima?

\section{Objetivos}

- Realizar el análisis psicométrico del Inventario de Dominancia cerebral.

- Realizar el análisis psicométrico del Cuestionario de autorreporte del desempeño laboral docente.

- Analizar las relaciones que existen entre la dominancia cerebral y el desempeño laboral de los docentes de una UGEL de Lima.

- Describir las diferencias que existen entre los docentes varones y mujeres de una UGEL de Lima respecto de las Escalas de dominancia cerebral.

- Describir las diferencias que existen entre los docentes varones y mujeres de una UGEL de Lima respecto de las Escalas de desempeño laboral.

- Establecer las diferencias que existen entre los docentes provenientes de universidades e institutos pedagógicos una UGEL de Lima respecto de las escalas de dominancia cerebral.

- Establecer las diferencias que existen entre los docentes provenientes de Universidades e institutos pedagogicos una UGEL de Lima respecto de las escalas de desempeño laboral.

- Identificar el tipo de dominancia cerebral predominante entre los docentes de una UGEL de Lima

- Identificar el cuadrante cerebral predominante entre los docentes de una UGEL de Lima 


\section{Hipótesis}

1. Existen relaciones significativas entre la dominancia cerebral y el desempeño laboral de los docentes de una UGEL de Lima

2. Existen diferencias significativas entre los docentes varones y mujeres de una UGEL de Lima, respecto de las Escalas de Dominancia Cerebral.

3. Existen diferencias significativas entre los docentes varones y mujeres de una UGEL de Lima, respecto de las Escalas de Desempeño Laboral.

4. Existen diferencias significativas entre los docentes provenientes de Universidades e institutos pedagogicos de una UGEL de Lima, respecto de las Escalas de dominancia cerebral.

5. Existen diferencias significativas entre los docentes provenientes de Universidades e institutos pedagogicos de una UGEL de Lima respecto de las escalas de desempeño laboral.

\section{MÉTODOS Y RESULTADOS}

El método de investigación utilizado fue el descriptivo, ya que permite describir las situaciones y eventos, además mide diversos aspectos del fenómeno a investigar. El estudio descriptivo se desarrolla describiendo las situaciones y eventos, es decir cómo se manifiesta el fenómeno a investigar, ya que éste busca especificar las propiedades importantes del problema en cuestión. El método descriptivo mide independientemente los conceptos y también pueden ofrecer la posibilidad de predicciones aunque sean muy rudimentarias (Sánchez y Reyes, 2003).

\section{Diseño de investigación}

Para la selección del diseño de investigación se ha utilizado como base el libro de Hernández, Fernández y Baptista (2006) titulado Metodología de la investigación. Según estos autores el diseño adecuado para esta investigación es de tipo descriptivo correlacional.

Su diagrama representativo es el siguiente:

\begin{tabular}{|cc|}
\hline & Ox \\
$M$ & $r$ \\
& Oy \\
\hline
\end{tabular}

En el esquema:

M = Muestra de investigación

Ox, Oy = Observaciones de las variables

$\mathbf{r} \quad=$ Relaciones entre variables

En este caso, se quiere correlacionar las variables Dominancia Cerebral y Desempeño laboral de los docentes de las Instituciones educativas de una UGEL de Lima. 


\section{Muestra}

La muestra de estudio que utilizamos, en tanto se ajusta a nuestras necesidades, es una muestra no probabilística de tipo intencionado en la medida que es el investigador quien ha determinado de manera intencional el lugar y la muestra con la que se trabajó. Esta decisión se tomó considerando que aplicar los instrumentos es una tarea muy complicada puesto que los docentes colaboran muy poco; sin embargo, creemos que la muestra tomada es bastante representativa.

Se tomó como muestra docentes de nueve instituciones educativas que en total suman 251, con edades que fluctúan entre 23 y 57 años de edad, presentando una predominancia los profesores cuyas edades están en los 30, 32, 36 y 45 años de edad. También se aprecia que la muestra está conformada mayoritariamente por mujeres $53 \%$, frente al $47 \%$ de los varones, igualmente de puede apreciar que los docentes provenientes de institutos pedagógicos son mayoritarios, $65,3 \%$ frente al $34,7 \%$ que proviene de las universidades.

Tabla N. ${ }^{0}$ 1. Composición general de la muestra.

\begin{tabular}{lcc}
\hline \multicolumn{1}{c}{ Sexo } & F & \% \\
\hline Masculino & 118 & 47.0 \\
Femenino & 133 & 53.0 \\
\hline Lugar de estudio & & \\
\hline Universidad & 87 & 34.7 \\
Instituto & 164 & 65.3 \\
\hline
\end{tabular}

\section{Instrumentos}

Los instrumentos utilizados en el desarrollo de la presente investigación fueron:

- El Instrumento de Dominancia Cerebral de Ruiz-Bolívar, B., Gardié, O., Ismael, A., Mendoza, Y. y Monasterios, G. que es una Adaptación del HBDI (Instrumento de Dominancia Cerebral de Herrmann). Este instrumento está compuesto por cuatro escalas independientes que evalúan el uso que habitualmente hacen los sujetos de su cerebro. Permite identificar el estilo preferencial del uso del pensamiento o de la forma cómo cada persona procesa la información en el cerebro.

- Cuestionario de autorreporte del desempeño laboral docente, elaborado por José Manuel Fernández Arata (2002). En la construcción de este instrumento se tomaron en cuenta las dimensiones propuestas por Valdés (2000):

- Capacidades pedagógicas.

- Emocionalidad.

- Responsabilidad en el desempeño de sus funciones laborales.

- Relaciones interpersonales con sus alumnos, padres, directivos, docentes y comunidad escolar en general.

- Resultados de su labor educativa. 
Los resultados son calificados sobre la base de una escala Likert de siete puntos, los cuales permitían expresar en términos de frecuencia la ocurrencia de los aspectos indicados y fluctuaban desde Nunca (1) para el menor valor hasta Siempre (7) para la mayor valoración.

\section{Análisis de validez y confiabilidad de los instrumentos}

En la medida que se necesita garantizar la idoneidad de los instrumentos de evaluación, se procedió a realizar los análisis estadísticos respectivos que nos indiquen sus niveles de validez y confiabilidad. El análisis psicométrico del inventario de dominancia cerebral indica que sus escalas alcanzan alfa de Cronbach que fluctúa entre 0.74 y 0.87 por lo que se considera que la prueba es confiable.

El análisis psicométrico del inventario de desempeño laboral docente indica que sus escalas alcanzan alfa de Cronbach que fluctúa entre 0.70 y 0.79 por lo que se considera que la prueba es confiable.

Los análisis de la Validez de los instrumentos realizados a través del Análisis factorial Exploratorio nos muestran que las pruebas presentan validez de Constructo.

\section{RESULTADOS}

Los resultados del análisis exploratorio de los datos (ver Tabla N. ${ }^{\circ}$ 2), en lo que se refiere a la forma de distribución, efectuado a través del test de bondad de ajuste a la curva normal de Kolmogorov-Smirnov, indican que en todas las variables estudiadas, se obtienen coeficientes K-S Z que no son estadísticamente significativos, por ello se puede concluir que se presentan adecuadas aproximaciones a la curva normal, por lo que es factible utilizar para el análisis de los datos, estadísticas paramétricas.

Tabla N. ${ }^{\circ}$ 2. Análisis de la Bondad de Ajuste de la Curva Normal de las Variables estudiadas.

\begin{tabular}{lccc}
\hline \multicolumn{1}{c}{ VARIABLE } & MEDIA & D.S. & K-S Z \\
\hline $\begin{array}{l}\text { Cuadrante A } \\
\begin{array}{l}\text { Superior izquierdo cerebral } \\
\text { Cuadrante B }\end{array}\end{array}$ & 36.61 & 4.57 & 1.17 \\
$\begin{array}{l}\text { Inferior izquierdo límbico } \\
\text { Cuadrante C }\end{array}$ & 38.72 & 4.23 & 1.21 \\
$\begin{array}{l}\text { Derecho inferior límbico } \\
\text { Cuadrante D }\end{array}$ & 37.51 & 4.10 & 1.26 \\
$\begin{array}{l}\text { Derecho superior cerebral } \\
\text { Desempeño en el aula }\end{array}$ & 35.89 & 4.33 & 1.15 \\
$\begin{array}{l}\text { Autoevaluación de las labores en el ámbito escolar } \\
\text { Interacción con los alumnos }\end{array}$ & 144.54 & 13.09 & 1.15 \\
\hline
\end{tabular}


El análisis de las correlaciones entre la dominancia cerebral y el desempeño docente indica que existen correlaciones significativas y positivas en todos los casos, con excepción de la Autoevaluación de las Labores en el Ámbito Escolar que no presenta correlación significativa alguna.

Tabla $N^{0}$ 3. Matriz de correlaciones entre los puntajes de los cuadrantes cerebrales y las escalas del desempeño laboral.

\begin{tabular}{|c|c|c|c|c|}
\hline Variables & $\begin{array}{l}\text { Desempeño } \\
\text { en el Aula }\end{array}$ & $\begin{array}{c}\text { Autoevaluación } \\
\text { Labores }\end{array}$ & $\begin{array}{l}\text { Interacción } \\
\text { Alumnos }\end{array}$ & $\begin{array}{l}\text { Desempeño } \\
\text { Total }\end{array}$ \\
\hline $\begin{array}{l}\text { Cuadrante A } \\
\text { Superior izquierdo cerebral }\end{array}$ & $0.32 * * *$ & -0.10 & $0.31 * * *$ & $0.22 * * *$ \\
\hline $\begin{array}{l}\text { Cuadrante B } \\
\text { Inferior izquierdo límbico }\end{array}$ & $0.47 * * *$ & -0.08 & $0.37 * * *$ & $0.34 * * *$ \\
\hline $\begin{array}{l}\text { Cuadrante } \mathrm{C} \\
\text { Derecho inferior límbico }\end{array}$ & $0.30 * * *$ & 0.04 & $0.32 * * *$ & $0.29 * * *$ \\
\hline $\begin{array}{l}\text { Cuadrante D } \\
\text { Derecho superior cerebral }\end{array}$ & $0.36 * * *$ & -0.01 & $0.39 * * *$ & $0.32 * * *$ \\
\hline
\end{tabular}

${ }^{* * *} p<.001$

$N=251$

El análisis comparativo de las Escalas de Dominancia cerebral por lugar donde estudió (ver Tabla N. ${ }^{\circ} 4$ ) indica que existen diferencias estadísticas significativas en el Cuadrante $\mathrm{B}$ - Inferior Izquierdo Límbico $(\mathrm{Z}=-2.13 \mathrm{p}<.05)$ y el Cuadrante $\mathrm{C}(\mathrm{Z}=-2.22 \mathrm{p}$ $<.05$ ), apreciándose en ambos casos que los profesores que estudiaron en un instituto presentan valores superiores a los profesores que estudiaron en la universidad.

Tabla N. ${ }^{\circ}$ 4. Prueba $\mathrm{Z}$ de comparación de medias de los puntajes de las Escalas de Dominancia Cerebral por lugar de estudio.

\begin{tabular}{|c|c|c|c|c|c|}
\hline \multirow[t]{2}{*}{ Variables } & \multicolumn{2}{|c|}{$\begin{array}{l}\text { Universidad } \\
\quad \mathbf{N}=\mathbf{8 7}\end{array}$} & \multicolumn{2}{|c|}{$\begin{array}{l}\text { Instituto } \\
\mathrm{N}=164\end{array}$} & \multirow[t]{2}{*}{$\mathbf{Z}$} \\
\hline & $\mathbf{M}$ & D.E. & $\mathbf{M}$ & D.E & \\
\hline $\begin{array}{l}\text { Cuadrante A } \\
\text { Superior izquierdo cerebral }\end{array}$ & 37.03 & 4.56 & 36.41 & 4.58 & 0.99 \\
\hline $\begin{array}{l}\text { Cuadrante B } \\
\text { Inferior izquierdo límbico }\end{array}$ & 37.89 & 3.89 & 39.09 & 4.33 & $-2.13 *$ \\
\hline $\begin{array}{l}\text { Cuadrante } \mathrm{C} \\
\text { Derecho lnferior límbico }\end{array}$ & 36.67 & 4.14 & 37.90 & 4.03 & $-2.22 *$ \\
\hline $\begin{array}{l}\text { Cuadrante D } \\
\text { Derecho superior cerebral }\end{array}$ & 35.53 & 4.55 & 36.06 & 4.23 & -0.90 \\
\hline
\end{tabular}

* $p<.05$

$n=251$ 
El análisis comparativo del desempeño por Lugar de estudio (ver tabla N. ${ }^{\circ}$ 5), efectuado a través de la prueba $\mathrm{Z}$ de diferencia de medias independientes, permite notar que no existen diferencias significativas en caso alguno.

Tabla N. ${ }^{0}$ 5. Prueba $\mathrm{Z}$ de comparación de medias de los puntajes de las Escalas de Desempeño Laboral por Lugar de estudio.

\begin{tabular}{|c|c|c|c|c|c|}
\hline \multirow[t]{2}{*}{ Variables } & \multicolumn{2}{|c|}{$\begin{array}{l}\text { Universidad } \\
\quad \mathbf{N}=\mathbf{8 7}\end{array}$} & \multicolumn{2}{|c|}{$\begin{array}{l}\text { Instituto } \\
N=164\end{array}$} & \multirow[t]{2}{*}{$\mathbf{Z}$} \\
\hline & $\mathbf{M}$ & D.E. & M & D.E & \\
\hline Desempeño en el aula & 145.49 & 12.52 & 142.47 & 14.11 & 1.08 \\
\hline $\begin{array}{l}\text { Autoevaluación de las labores en el } \\
\text { ámbito escolar }\end{array}$ & 62.82 & 11.63 & 62.01 & 10.57 & 0.53 \\
\hline Interacción con los alumnos & 35.87 & 4.17 & 35.80 & 5.07 & 0.12 \\
\hline Desempeño total & 244.19 & 19.19 & 240.28 & 21.41 & 1.44 \\
\hline
\end{tabular}

$n=251$

El análisis comparativo de las Escalas de Dominancia cerebral por sexo presentado en la Tabla N. ${ }^{\circ} 6$, realizado a través de la prueba $\mathrm{Z}$ de diferencia de medias independientes, permite notar que no existen diferencias por Sexo.

Tabla N. ${ }^{\circ}$ 6. Prueba $\mathrm{Z}$ de comparación de medias de los puntajes de las Escalas de Dominancia Cerebral por sexo.

\begin{tabular}{lccccc}
\hline \multirow{2}{*}{ Variables } & \multicolumn{2}{c}{ Varón } & \multicolumn{2}{c}{ Mujer } & \multirow{2}{*}{$\mathbf{N = \mathbf { 1 3 3 }}$} \\
\cline { 2 - 5 } & $\mathbf{M}$ & D.E. & $\mathbf{M}$ & $\mathbf{Z}$ & $\mathbf{Z}$ \\
\cline { 2 - 5 } Cuadrante A & 37.19 & 4.44 & 36.08 & 4.64 & 1.93 \\
Cuadrante B & 39.17 & 4.08 & 38.30 & 4.32 & 1.63 \\
Cuadrante C & 37.18 & 3.96 & 37.80 & 4.20 & -1.19 \\
Cuadrante D & 35.93 & 4.11 & 35.85 & 4.52 & 0.13 \\
\hline${ }^{*} \mathrm{p}<.05$ & & & & &
\end{tabular}


El análisis comparativo del desempeño laboral por sexo (ver tabla $\mathrm{N}^{0} 7$ ), efectuado a través de la prueba $\mathrm{Z}$ de diferencia de medias independientes, permite notar que no existen diferencias significativas en caso alguno.

Tabla N. ${ }^{\circ}$ 7. Prueba $Z$ de comparación de medias de los puntajes de las Escalas de Desempeño Laboral por sexo.

\begin{tabular}{|c|c|c|c|c|c|}
\hline \multirow{2}{*}{ Variables } & \multicolumn{2}{|c|}{$\begin{array}{c}\text { Varón } \\
\mathrm{N}=118\end{array}$} & \multicolumn{2}{|c|}{$\begin{array}{c}\text { Mujer } \\
\mathbf{N}=133\end{array}$} & \multirow[t]{2}{*}{$\mathbf{Z}$} \\
\hline & $\mathbf{M}$ & D.E. & $\mathbf{M}$ & D.E & \\
\hline Desempeño en el aula & 145.38 & 12.71 & 143.13 & 13.36 & 0.96 \\
\hline $\begin{array}{l}\text { Autoevaluación de las labores en el } \\
\text { ámbito escolar }\end{array}$ & 61.51 & 10.56 & 63.50 & 11.83 & -1.40 \\
\hline Interacción con los alumnos & 36.37 & 4.52 & 35.38 & 4.37 & 1.76 \\
\hline Desempeño total & 243.26 & 19.19 & 242.68 & 20.68 & 0.23 \\
\hline
\end{tabular}

* $p<.05$

$n=251$

Los resultados obtenidos en la Tabla N. ${ }^{\circ} 8$ nos indican que los profesores sin dominancia cerebral son significativamente mayores al alcanzar el $43.4 \%$ de los casos, luego siguen los profesores que tienen dominancia simple con el $21.9 \%$. Sólo el $11.2 \%$ de los profesores estaría utilizando el total de su cerebro al tener dominancia cuádruple.

Tabla N. ${ }^{0}$ 8. Análisis del Tipo de Dominancia cerebral.

\begin{tabular}{lcc}
\hline Tipo & Frecuencia & Porcentaje \\
\hline Simple & 55 & 21.9 \\
Doble & 38 & 15.1 \\
Triple & 21 & 8.4 \\
Cuádruple & 28 & 11.2 \\
Sin dominancia & 109 & 43.4 \\
\hline Total & 251 & 100.0 \\
\hline $\mathrm{n}=251$ & &
\end{tabular}

Los resultados obtenidos en la Tabla N. 9 nos indican que en los profesores de la muestra predomina el cuadrante $\mathrm{B}$ (destinado a cumplir programaciones previamente planificadas sin ánimos de cambio), seguido por el cuadrante $\mathrm{C}$ (emocional), es decir, los cuadrantes del cerebro límbico; luego vienen el cuadrante A y el cuadrante D, pertenecientes al cerebro conceptual y lógico, es decir aquellos relacionados a la producción de conocimientos. 
Tabla N. ${ }^{\circ}$ 9. Análisis jerárquico de la Dominancia cerebral

\begin{tabular}{lccc}
\hline VARIABLE & MEDIA & D. E. & PUESTO \\
\hline $\begin{array}{l}\text { Cuadrante B } \\
\text { Inferior izquierdo límbico }\end{array}$ & 38.72 & 4.23 & $1^{\circ}$ \\
$\begin{array}{l}\text { Cuadrante C } \\
\text { Derecho inferior límbico }\end{array}$ & 37.51 & 4.10 & $2^{\circ}$ \\
$\begin{array}{l}\text { Cuadrante A } \\
\text { Superior izquierdo cerebral }\end{array}$ & 36.61 & 4.57 & $3^{\circ}$ \\
$\begin{array}{l}\text { Cuadrante D } \\
\text { Derecho superior cerebral }\end{array}$ & 35.89 & 4.33 & $4^{\circ}$ \\
\hline
\end{tabular}

$n=251$

\section{ANÁLISIS Y DISCUSIÓN}

Considerando los resultados del análisis psicométrico de la prueba de modos de pensamiento de los hemisferios cerebrales, observamos que todos los ítems forman parte de dicha prueba y corresponden a cada una de las escalas asignadas por Herrmann. El instrumento es confiable en la medida que sus escalas obtuvieron coeficientes Alfa de Cronbach con valores que oscilan entre 0.74 y 0.84 .

En cuanto a la validez, el resultado del Análisis Factorial Exploratorio indica que la prueba está conformada por un solo factor que explica el $78.43 \%$ de la varianza total. Estos hallazgos coinciden con lo propuesto teóricamente por Herrmann, y con lo reportado en otros estudios con muestras diferentes, como por ejemplo Ruiz-Bolívar, B., Gardié, O., Ismael, A., Mendoza, Y. y Monasterios, G. (1994).

En cuanto al Inventario de desempeño laboral docente, los análisis psicométricos indican que todos los ítemes son confiables y forman parte de dicho cuestionario. Las escalas obtienen coeficientes alfa de Cronbach con valores que oscilan entre 0.74 y 0.87 . El instrumento es confiable en la medida que el análisis generalizado de la prueba arroja un alfa de Cronbach de 0.83 .

Los resultados del Análisis Factorial Exploratorio del Inventario de desempeño laboral docente indican que está conformada por un factor, agrupado en tres escalas, y que explica el $63.20 \%$ de la varianza total. Los resultados permiten concluir que el Inventario de desempeño laboral docente presenta validez de Constructo. Estos hallazgos coinciden con lo propuesto teóricamente por Valdez Veloz (2000) y que sirvió de base para la elaboración de la prueba; igualmente, estos resultados coinciden con los obtenidos por el autor de la prueba Manuel Fernández (2002).

Los resultados en cuanto a las relaciones de la dominancia cerebral con el desempeño laboral de los docentes de una UGEL de Lima (Tabla N. ${ }^{\circ} 3$ ) indican que se acepta en lo fundamental la primera hipótesis de investigación. Se observa que existen correlaciones significativas y positivas en todos los casos, a excepción de la Autoevaluación de las Labores 
en el Ámbito Escolar. Estos resultados, que en general son valores moderados, nos hacen pensar que los docentes podrían no estar vinculando con la fortaleza necesaria sus funciones cerebrales en su trabajo docente. En este aspecto se coincide con los resultados encontrados por Gardie (1999), quien afirma que los docentes dan prioridad al cumplimiento de programas, rutinas y seguimiento de pautas pre-establecidas, sobre otras formas de pensamiento relacionadas con la producción rigurosa de conocimientos, la proposición de ideas novedosas, etc.

A partir de estos resultados, podemos resaltar la urgencia de que los profesores tomen conciencia de la necesidad de conocer más sobre el cerebro y de que manejen más información sobre cómo funciona este órgano para que así desarrollen una enseñanza, un ambiente escolar, un currículo, una evaluación más acordes con las características intrínsecas e innatas de nuestros cerebros para aprender o, en otras palabras, más compatibles con la manera cómo aprende nuestro cerebro.

En cuanto al análisis de las diferencias entre los grupos establecidos y correspondientes a las hipótesis $\mathrm{N} .{ }^{\text {os }} 2,3,4$ y 5 , los resultados muestran que solo existen diferencias significativas en el caso del Cuadrante B - Inferior izquierdo límbico y el Cuadrante C, apreciándose en ambos casos que los profesores que estudiaron en un instituto presentan valores superiores a los profesores que estudiaron en la universidad. Estos resultados nos indicarían que los problemas en la utilización del cerebro conceptual se agudizan entre los docentes provenientes de los institutos.

En lo que se refiere a los tipos de pensamiento predominantes entre los docentes de la muestra, encontramos que un porcentaje significativamente alto de docentes (43.4\%) no tiene dominancia cerebral establecida, es decir su funcionamiento cerebral no tiene la intensidad ni la amplitud que debería tener dada su condición de maestro. Sin embargo, es necesario anotar que un porcentaje relativamente bajo, pero significativo de docentes (11.2\%), trata de funcionar con todo su cerebro lo que nos debe llevar a insistir en la necesidad de preparar adecuadamente a los maestros en tanto son ellos quienes tienen directa participación en el desarrollo de las funciones cognitivas de los alumnos.

En lo referente al análisis jerárquico de la dominancia cerebral de los docentes (Tabla N. ${ }^{\circ}$ 9), el cuadrante predominante es el B (38.72); este resultado coincide con el encontrado por Gardié (1999) en docentes venezolanos. Estos resultados deben llevarnos a la reflexión habida cuenta que nuestros docentes deberían ser personas con dominancia cerebral cortical pues ellos tienen una inteligencia sólida y viva, es un técnico de alto nivel, dotado de un excelente espíritu de análisis y de síntesis, está bien situado para triunfar en la sociedad actual.

\section{CONCLUSIONES}

1. El Inventario de Dominancia cerebral ha demostrado ser un instrumento válido y confiable al ser aplicado en docentes de instituciones educativas, puede ser utilizado en posteriores investigaciones.

2. El Cuestionario de autorreporte del desempeño laboral docente, conformado por 47 ítemes, ha demostrado que sus tres escalas son confiables y válidas en docentes de instituciones educativas. 
3. El análisis de los resultados muestran que existen relaciones significativas entre la dominancia cerebral y el desempeño laboral de los docentes de una UGEL de Lima, salvo el caso de la escala de autoevaluación de las labores en el ámbito escolar.

4. Los resultados alcanzados nos muestran que no existen diferencias entre los docentes varones y mujeres de una UGEL de Lima, respecto de las Escalas de dominancia cerebral.

5. Los resultados alcanzados nos muestran que no existen diferencias entre los docentes varones y mujeres de una UGEL de Lima respecto de las Escalas de desempeño laboral.

6. El análisis de los resultados muestran que existen diferencias significativas solo en los casos del Cuadrante B y el Cuadrante C, apreciándose en ambos casos que los profesores que estudiaron en un instituto presentan valores superiores a los profesores que estudiaron en la universidad.

7. El análisis de los resultados muestran que no existen diferencias significativas entre los docentes provenientes de Universidades e institutos pedagogicos de una UGEL de Lima respecto de las Escalas de desempeño laboral.

8. Los análisis estadísticos realizados nos indican que no existe un tipo de dominancia cerebral predominante entre los docentes de una UGEL de Lima.

9. Los análisis estadísticos realizados nos indican que el cuadrante cerebral predominante entre los docentes de una UGEL de Lima es el cuadrante B.

\section{REFERENCIAS BIBLIOGRÁFICAS}

1. Austin, E. (1997). Las tres caras de la mente. Caracas: Editorial Galac, S. A.

2. Carter, R. (1998). El nuevo mapa del cerebro. Barcelona: Ediciones de la Librería.

3. Carretero, M. (1996). Constructivismo y problemas educativos: una relación compleja. Anuario de Psicología. España.

4. Díaz, H. y Saavedra, J. (2000). "La carrera de maestro: Factores institucionales, incentivos económicos y desempeño". Research Network Working Paper R-410. Washington D.C.: Banco Interamericano de Desarrollo.

5. Gazzaniga, M. (1977). Review of the split brain. En M.C. Wittrock (Ed.) The Human brain. Englewood Cliffs: Prentice-Hall, Inc.

6. Gutierrez, H. (2002). La evaluación como experiencia total. Logros, objetivos, procesos, competencia y desempeño. Colombia: Editorial Nomos S.A., Cooperativa Editorial Magisterio.

7. Guyton, A. (2006). Tratado de fisiología médica. México: Edit. Interamericana.

8. Hernández, Fernández y Baptista. (2006). Metodología de la investigación. México: Edit. Mc Graw Hill. 
9. Luria, A. (1985). Las funciones corticales superiores en el hombre. La Habana: Edit. Científico Técnico.

10. Ruiz-Bolívar, B.: Gardié, O.: Ismael, A.; Mendoza, Y.; Monasterios, G., y Richter. (1994). Adaptación y validación de la encuesta de HBDI para evaluar la dominancia cerebral: Un estudio preliminar. Colecciones CIEAPRO N. ${ }^{\circ}$ 1: 23-46.

11. Sánchez, H y Reyes, C. (2002).Metodología y diseños en la investigación científica. Lima: Universidad Ricardo Palma, Editorial Universitaria.

12. Ver Lee, W. (1986). Aprender con todo el cerebro. Barcelona (España): MartínezRoca.

13. Wittrock, M.C. (1977). The human brain. Englewood Cliffs (New Jersey): PrenticeHall, Inc. 